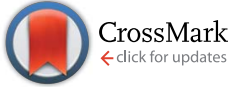

Cite this: RSC Adv., 2016, 6, 80700

Received 27th June 2016

Accepted 18th August 2016

DOI: $10.1039 /$ c6ra16560e

www.rsc.org/advances

\section{Three-dimensional morphology of the interface between micro porous layer and catalyst layer in a polymer electrolyte membrane fuel cell $\dagger$}

\begin{abstract}
L. Zielke, ${ }^{a}$ S. Vierrath, ${ }^{a}$ R. Moroni, ${ }^{a}$ A. Mondon, ${ }^{c}$ R. Zengerle ${ }^{a}$ and S. Thiele ${ }^{\star a b}$
Interfaces between the different layers in proton exchange membrane fuel cells are expected to influence transport properties and therefore cell performance. So far the interface between micro porous layer (MPL) and catalyst layer (CL) has been difficult to investigate due to its nanometer scale morphology. We apply focused ion beam scanning electron microscopy tomography with pore contrasting via atomic layer deposition to reconstruct a representative volume of $5.1 \mu \mathrm{m} \times 1.5 \mu \mathrm{m} \times 4.5 \mu \mathrm{m}$ containing CL, MPL and their interface. We find that platinum in the CL results in brighter SEM image intensities, compared to the MPL. This allows (i) estimating the extension of the interfacial region $(530 \mathrm{~nm})$, (ii) evaluating Pt-content homogeneity in the $C L$ and (iii) calculating the individual roughnesses for the $C L(102 \mathrm{~nm})$ and for the MPL (129 nm). We further calculate porosity, pore sizes, and oxygen diffusivities. Thus, we find that the values of the parameters of the interfacial region are between those of the $C L$ and the MPL, meaning that on the investigated scale, the interface is a homogeneous transitional region. A representativeness analysis shows that our reconstructed volume is sufficiently large concerning all calculated parameters.
\end{abstract}

\section{Introduction}

Fuel cells consist of multiple porous layers: the proton exchange membrane (PEM), the catalyst layers (CLs), the micro porous layers (MPLs) and gas diffusion layers (GDLs). Since these layers are sandwiched, not only the layers themselves but also their interfaces influence the performance of the fuel cell. ${ }^{\mathbf{1}}$ It is therefore crucial to know the morphology of the interfaces, especially between the CL and the MPL, where most transported species have to pass in order to reach their destinations within the fuel cell (see Fig. 1a). ${ }^{1-4}$ The fabrication method of the fuel cell has substantial impact on the morphology of the layers and the corresponding interfaces. ${ }^{5}$ For bringing CLs and MPLs in contact, hot-pressing (catalyst coated membrane, CCM) and spray-coating (gas diffusion electrode, GDE) are the two most common assembly methods. ${ }^{6}$ The major differences between these methods are described in the ESI and are illustrated in Fig. S1.†

\footnotetext{
${ }^{a}$ Laboratory for MEMS Applications, IMTEK, Department of Microsystems Engineering, University of Freiburg, Georges-Koehler-Allee 103, 79110 Freiburg, Germany.E-mail: lukas.zielke@imtek.de; severin.vierrath@imtek.de; riko.moroni@ imtek.de; zengerle@imtek.uni-freiburg.de; simon.thiele@imtek.de

${ }^{b}$ FIT, University of Freiburg, Georges-Koehler-Allee 105, 79110 Freiburg, Germany ${ }^{c}$ Fraunhofer Institute for Solar Energy Systems ISE, Division Energy Technologies, Heidenhofstr. 2, 79110 Freiburg, Germany. E-mail: andrew.mondon@ise.fraunhofer. de

† Electronic supplementary information (ESI) available. See DOI: 10.1039/c6ra16560e
}

In order to obtain information about transport and morphology, a three-dimensional reconstruction of the interfacial region (IR) is needed for CCMs as well as for GDEs. Such a three-dimensional dataset of an interface can be used to either validate modelling assumptions ${ }^{7}$ or to interpret experimental results. ${ }^{8}$ Several reconstructions showing exclusively the $\mathrm{CL}^{9-15}$ or the $\mathrm{MPL}^{\mathbf{1 6 - 2 0}}$ are available in literature where the individual roughness $^{6}$ of CL and MPL at the interface has been measured ${ }^{4}$ and used for virtual reconstruction of a CCM-GDL sandwich. ${ }^{1}$ In a recent study X-ray tomography with a voxel volume of $(2 \mu \mathrm{m})^{3}$ was employed in order to visualize gaps between CL and MPL in a CCM-GDL sandwich. ${ }^{21}$ The roughness measurements in $^{4}$ suggest that the interface between the CL and the MPL exhibits large gaps that originate from pressing the two rough surfaces onto each other which was confirmed by. ${ }^{21}$ In these large pores liquid water is expected to accumulate., ${ }^{\mathbf{4 , 2 2}}$ However, the surface investigation methods are not able to image the interface in the actual application-relevant state, i.e. the final sandwich (see Fig. 1a and b). While X-ray tomography is indeed capable of imaging the interface in the assembled state, the resolution is not sufficient for imaging the morphology in the range of the carbon particles in the CL and MPL $(\sim 40 \mathrm{~nm})$. As GDEs recently gained importance due to record power densities of fuel cell fabricated with GDEs and directly deposited membranes, ${ }^{23-25}$ we extend the knowledge of CL/MPL interfaces in GDEs by applying a combination of atomic layer deposition (ALD) and focused ion beam scanning electron microscopy tomography (FIB-SEM tomography). This approach allows to image and analyse the interface between assembled CL and MPL in a volume of $5.1 \mu \mathrm{m}$ 
a

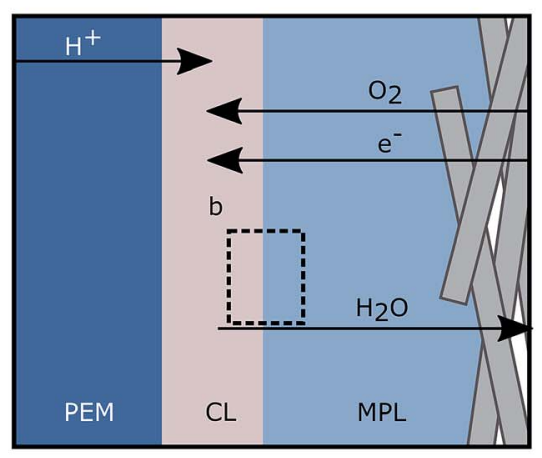

b

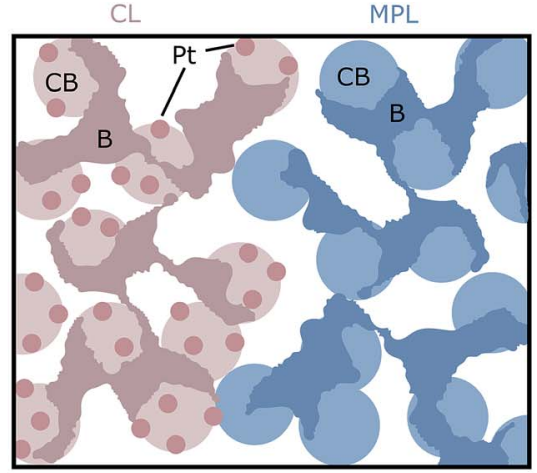

c

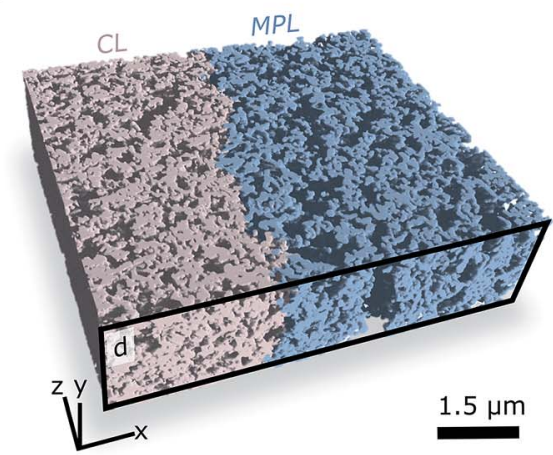

d

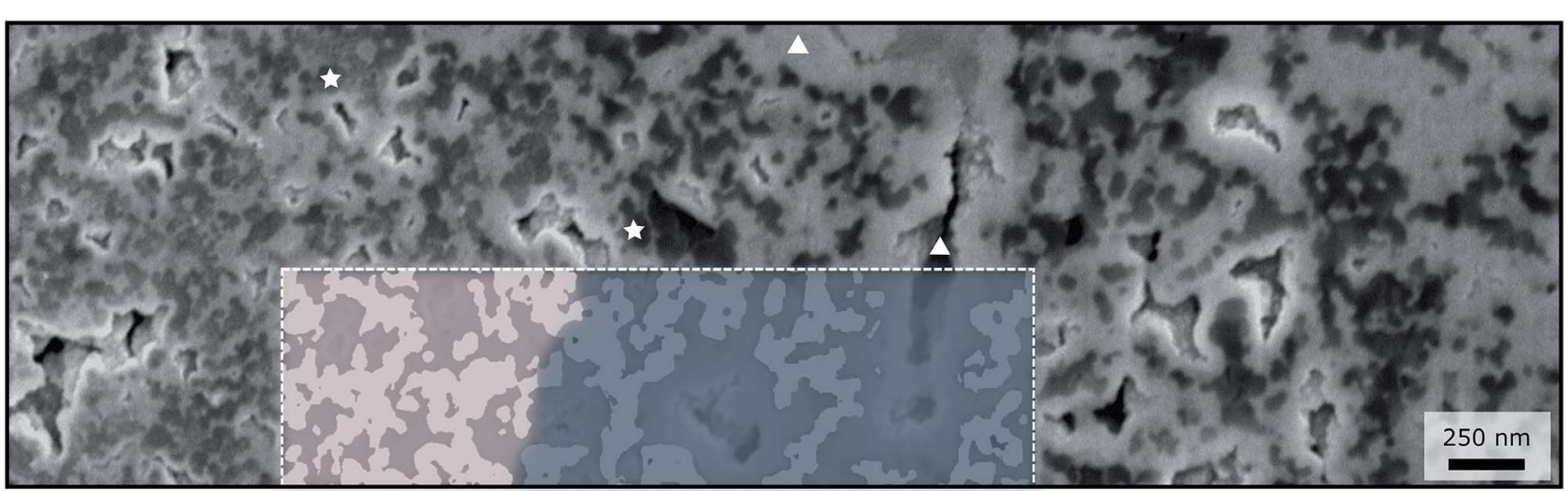

Fig. 1 Schematic representation and reconstruction of the interface between CL and MPL. (a) Cathode side of a fuel cell: CL (rose), MPL (light blue), GDL (grey), PEM (dark blue), with the transported species shown as arrows. (b) Schematic structure of the interface between CL and MPL. The $C L$ is loaded with Pt particles. (c) Three-dimensional reconstruction of the CL-MPL interfacial area using FIB-SEM tomography. (d) $X-Y$ plane (SEM imaging plane) of the reconstruction. The dark region (right) is the MPL and the bright region the CL (left). Stars and triangle exemplarily represent solid material and pores respectively. A part of the corresponding segmentation is shown in the white dotted inset.

$\times 1.5 \mu \mathrm{m} \times 4.5 \mu \mathrm{m}$ with voxel volumes of $(3 \mathrm{~nm})^{3}$. Thus the morphology in the range of the carbon particles can be examined and the interface generated by spray-coating the CL onto the MPL investigated. In this study we use a conditioned GDE, serving as a baseline for future investigations.

FIB-SEM tomography is a prominent and well known method in materials science to reconstruct sample volumes with up to $40 \mu \mathrm{m}$ side length. ${ }^{26}$ In order to investigate a large quantity of samples in a reasonable amount of time using FIBSEM tomography, highly porous samples must be infiltrated. ${ }^{27}$ We have recently shown a novel infiltration method on a CL reconstruction, where we used ALD as infiltration method. ${ }^{28}$ ALD infiltration is not limited to CLs. We have proven its suitability for other carbon based porous materials, e.g. carbon binder domains in batteries..$^{29}$ By employing ZnO ALD, a high contrast between pores, carbon and Pt particle loaded carbon is achieved. Additionally a large number of samples can be infiltrated simultaneously. By employing ALD infiltration and FIBSEM tomography, producing datasets with several $\mu \mathrm{m}$ edge lengths and a resolution of $3 \mathrm{~nm}$ is feasible. Since these settings allow investigating porous carbon structures on the scale of the carbon structures themselves ( $40 \mathrm{~nm}$ carbon spheres, $\sim 100 \mathrm{~nm}$ pores), in the application-relevant assembly and additionally in a large volume, this method was used for the reconstruction of a spray-coated CL/MPL interface.

\section{Experimental section}

\section{Sample preparation and infiltration}

The investigated sample was prepared by coating gas diffusion electrodes (Paxitech SAS, $0.5 \mathrm{mg} \mathrm{Pt} \mathrm{cm}^{-2}, 70 \% \mathrm{Pt} / \mathrm{C}$ ) with directly deposited membranes as described in. ${ }^{23}$ The coated electrodes were then assembled in a fuel cell and equilibrated at $80{ }^{\circ} \mathrm{C}, 90 \%$ relative humidity, ambient pressure with 0.251 $\mathrm{min}^{-1}$ hydrogen and $0.5 \mathrm{l} \mathrm{min}^{-1}$ oxygen. To access the interface of the catalyst layer and the microporous layer, the membrane electrode assembly was nitrogen freeze fractured. To ensure clean broken edges, the carbon fibres of the gas diffusion layer were gently peeled off in beforehand. The directly deposited membrane, being very well attached, served as a support for the remaining fragile layers. The excavated interface was then coated using ALD with a $100 \mathrm{~nm} \mathrm{ZnO}$ film by cyclic application of diethyl zinc and water at $50{ }^{\circ} \mathrm{C}$ in a vertical-flow, hot-wall reactor (OpAL, manufactured by Oxford Instruments) as described in ref. 30 .

\section{FIB/SEM tomography}

FIB-SEM tomography was made using a Zeiss Auriga 60 dual beam comprising 676 FIB tomography slices. Tomography was conducted at $30 \mathrm{kV}$ accelerating voltage and $20 \mathrm{pA}$ beam current with a slice thickness of $9 \mathrm{~nm}$. SEM images were 
acquired at $5 \mathrm{kV}$ and a pixel size of $3 \mathrm{~nm}$ using an inlens (IL) and a secondary electron detector (SE2). For sample surface protection, a Pt layer was deposited on top of the sampling volume. Subsequently lines were engraved to render slice thickness checking possible. The total reconstructed volume was $5.1 \mu \mathrm{m} \times 1.5 \mu \mathrm{m} \times 4.5 \mu \mathrm{m}$.

\section{Image processing and calculations}

Images were aligned using self-programmed MATLAB (Mathworks) ${ }^{31}$ functions. Image enhancement and segmentation were conducted using Fiji: ${ }^{32}$ first the grey value histograms were adjusted using a quantile based normalization, then threedimensional statistical region merging was applied. The resulting images were binarized using threshold according to visual judgment. After removing unconnected solid parts (island filtering) and manual correction, the sampling volume was divided into three sub-regions to facilitate individual fine corrections. To segment the interfacial region (IR), we applied a large minimum filter and multiplied the result with the segmented dataset. It was thus possible to discriminate clearly between dark regions (MPL) and bright regions (CL) in the interfacial region. The images of the SE2 and the IL detector showed a good contrast between the Pt particles loaded CL and the MPL. We quantified this contrast by calculating the difference of mean grey value of the CL and MPL for both detectors divided by the maximum intensity. Results are presented in the results section. The SE2 images lack contrast between $\mathrm{ZnO}$ and Pt particle loaded carbon, which was the reason that we mainly used the IL images for segmentation. Size distributions were determined based on the method presented by Delerue et al. ${ }^{33}$ implemented in MATLAB. $\mathrm{O}_{2}$ diffusivities were calculated using the Bosanquet solver implemented in the finite element solver GeoDict. ${ }^{34}$ The solver calculates the Knudsen (through random walk) and bulk diffusivity (by solving for Laplace's equation) in the pore space of the FIB/SEM reconstruction. Local porosities and the roughness were calculated using MATLAB. The calculated roughness corresponds to the arithmetic mean value of the distances from a surface point to a plane, fitted to the individual surfaces.

\section{Results and discussion}

The present reconstruction is $5.1 \mu \mathrm{m} \times 1.5 \mu \mathrm{m} \times 4.5 \mu \mathrm{m}$ large (Fig. 1c), with a voxel volume of $(3 \mathrm{~nm})^{3}$ and it contains part of the CL, a mixed CL/MPL region denoted interfacial region (IR), and part of the MPL. With the described approach we are able to differentiate between CL, MPL and pore space.

\section{Grey values and pore sizes}

As can be seen in Fig. 1d, the ALD infiltration technique facilitates the discrimination between pores (triangles mark bright $\mathrm{ZnO}$ areas and insufficiently filled pores) and solid (stars mark darker grey areas). The insufficiently filled pores are deleted using island filters accompanied by manual assessment of the filter decisions. A differentiation between CL (left in Fig. 1d) and MPL (right) is also possible, since the CL appears brighter in the SEM images due to the presence of Pt particles, which feature greater secondary electron yield than carbon only.

This is quantified in Fig. 2a, where the mean grey value (from 0 to 255) is shown. The mean grey value is averaged in the $y$-and $z$-direction, resulting in a mean grey value across the interface. The dependence of grey value and Pt-loading is first used to estimate the spatial extension of the IR (white region in Fig. 2a): the IR is $530 \mathrm{~nm}$ thick. Secondly, the mean grey value across the interface can be used to verify the homogeneity of the Ptdistribution. Overall the Pt-distribution appears homogeneous. However the two detector images give slightly different information: while the SE2 images suggest a homogeneous Ptdistribution along the CL, the mean grey value from the IL images shows that the Pt-content decreases when approaching the IR, with a $10 \%$ decrease in mean grey value. As the secondary electrons are guided through an electromagnetic lens system into the IL detector, only the SE2 detector images are considered for quantitative results, yielding a homogeneous Ptparticle distribution.

From the mean grey values, the contrast of CL and MPL can be quantified and compared. We find that the contrast in the SE2 detector images (0.13) is slightly better than in the IL detector images (0.11). However, the $\mathrm{ZnO}-\mathrm{CL}$ contrast is better
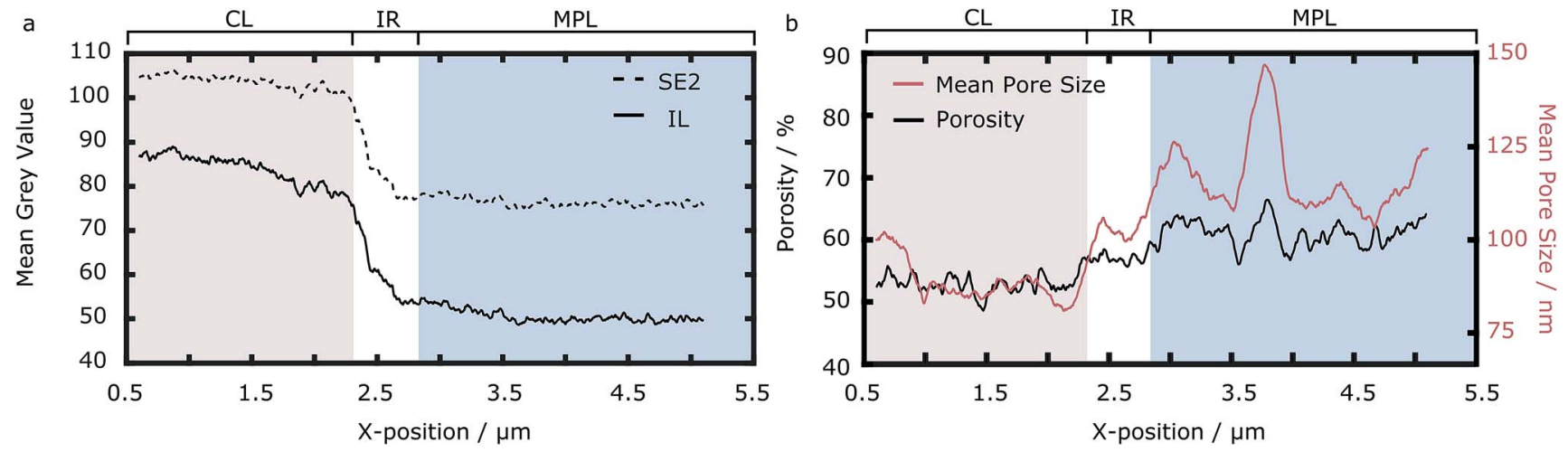

Fig. 2 (a) Mean grey value versus $x$-position of the reconstruction. (b) Mean porosity (black line) and mean pore sizes (red line) versus $x$-position of the reconstruction. 
in the IL images, which is the reason for using these images for reconstruction.

To quantify the morphology of the IR with respect to the CL and the MPL, we calculate porosity and mean pore size (diameter) across the interface. The global porosities of the three regimes are $53 \%$ for the $\mathrm{CL}, 56 \%$ for the interfacial region and $60 \%$ for the MPL. The local porosities and pore sizes across the interface are shown in Fig. 2b. The three regimes differed in mean porosity and mean pore sizes. The IR showed a larger porosity and mean pore size than the CL but lower values than the MPL. The IR also showed the smallest variance in porosity, suggesting a rather homogeneous morphology on the investigated length scale. The plots also reveal large cavities in the MPL increasing the local porosity and local mean pore size strongly as expected from MPLs. ${ }^{16}$ The most important result is that the IR does not show any significantly larger pores than the MPL in the investigated region.

\section{Roughness}

As mentioned before, the Pt content of the CL allows distinguishing between CL and MPL. With this information at hand the roughness of CL and MPL can be evaluated separately. As a measure of roughness we use the arithmetic mean value of the absolute distance value between surface points to a plane fitted through all surface points. The plane is fitted through this point cloud with a least square fit using MATLAB. This plane represents the base plane, as in optical profilometry, which then allows using the average roughness definition from British standards. ${ }^{35}$ We thereby find that CL and MPL have average roughnesses of $102 \mathrm{~nm}$ and $129 \mathrm{~nm}$ respectively. These values are not comparable with roughnesses from literature, which are commonly calculated or measured on a much larger scale (e.g. for the MPL $5.35 \mu \mathrm{m}^{22}$ ), where CL and MPL exhibit significant cracks and holes increasing the roughness drastically. ${ }^{\mathbf{4 , 2 1}}$ In fact, the found nano-roughness is complementary to larger scale roughnesses and can therefore be used in combination.

\section{Oxygen diffusivity}

In order to quantify the transport behaviour, we calculate $\mathrm{O}_{2}$ diffusivity in all three main directions for each region separately (MPL, IR, CL), for a combination of CL and MPL only (CL + MPL) and for the entire reconstruction (CL $+\mathrm{IR}+\mathrm{MPL})$. This allows evaluating the impact of the IR on diffusion across the layers. The calculated diffusion coefficients are shown in Table 1. We found that the IR itself does not significantly influence the diffusivity, especially in the direction where molecules have to pass the IR ( $x$-direction): the dataset with a removed IR (CL + MPL) exhibits a very similar diffusion coefficient, $1.87 \times 10^{-6}$ $\mathrm{m}^{2} \mathrm{~s}^{-1}$, compared to the coefficient of the entire dataset (CL + IR + MPL) $1.89 \times 10^{-6} \mathrm{~m}^{2} \mathrm{~s}^{-1}$. As the calculation of transport parameters should not depend on the size of the underlying reconstruction, we perform a representativeness analysis. That is, calculating the transport parameters for increasing subvolumes. The results are shown in Fig. 3.

Table $1 \mathrm{O}_{2}$ Bosanquet diffusivities for $\mathrm{CL}, \mathrm{MPL}, \mathrm{CL}+\mathrm{MPL}$ and for the entire dataset (CL + IR + MPL). The bulk diffusivity in the simulation was $2.09 \times 10^{-5} \mathrm{~m}^{2} \mathrm{~s}^{-1}$

\begin{tabular}{|c|c|c|c|c|c|c|c|c|c|c|c|c|c|c|c|}
\hline & \multicolumn{3}{|l|}{ CAT } & \multicolumn{3}{|l|}{ IR } & \multicolumn{3}{|l|}{ MPL } & \multicolumn{3}{|c|}{$\mathrm{CL}+\mathrm{MPL}$} & \multicolumn{3}{|c|}{$\mathrm{CL}+\mathrm{IR}+\mathrm{MPL}$} \\
\hline & $x$ & $y$ & $z$ & $x$ & $y$ & $z$ & $x$ & $y$ & $z$ & $x$ & $y$ & $z$ & $x$ & $y$ & $z$ \\
\hline
\end{tabular}

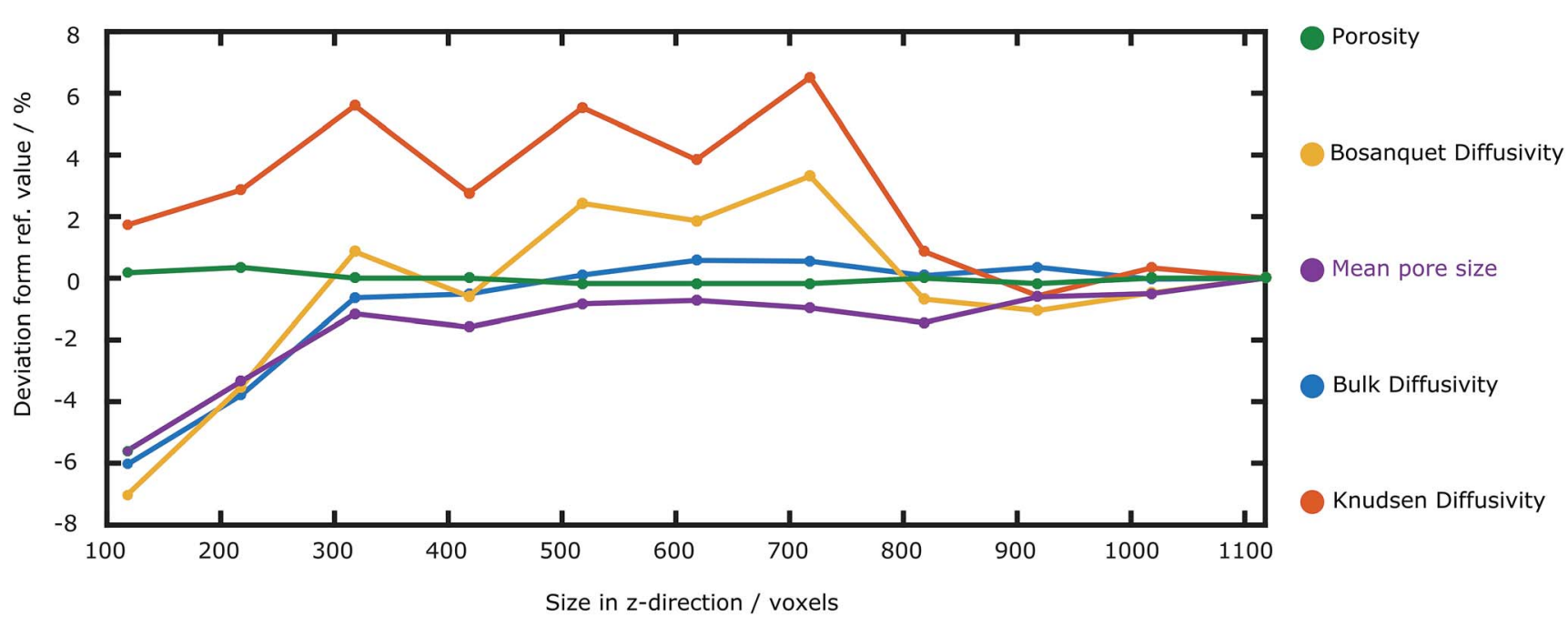

Fig. 3 Dependence of the calculated transport parameters on the size in z-direction of the reconstruction. All calculated parameters converge, however at different sizes in $z$-direction. 


\section{Representativeness}

In order to study how the calculated parameters depend on the size of the reconstructed volume, we calculate the Knudsen, bulk and Bosanquet diffusivities, the porosity and the mean pore size for differently sized subvolumes of the reconstruction. The deviation from the reference value (the value of the entire reconstruction) is plotted against the size in $z$-direction, along the IR. The bulk diffusivity converges for $z$-sizes larger than 300 voxels, which is also the case for the mean pore size. The Knudsen diffusivity, and thus also the Bosanquet diffusivity, however started converging at a $z$-size value of 800 voxels. The porosity hardly changes throughout the whole range of reconstruction sizes.

These results show that representativeness strongly depends on the calculated transport parameter. It is thus necessary to conduct such an analysis when calculating different parameters. The results here imply that our reconstruction is large enough for feasible characterization of the pore morphology and transport through the interfacial region.

\section{Conclusions}

We successfully employ ALD infiltration and subsequent FIBSEM tomography on a CL/MPL interface of a gas diffusion electrode. We are able to discriminate not only between pore and solid, but also between CL and MPL due to the Pt particle loading of the CL and resulting image contrast in the electron microscope images. We calculate $\mathrm{O}_{2}$ diffusivity and find that the interfacial region does not differ significantly from the CL or MPL and is a homogeneous transitional region between CL and MPL. We further check and approve representativeness for all calculated parameters. For future studies we strongly recommend the use of both SEM detectors: the inlens (IL) detector exhibits high contrast between pores and solid, while the contrast between MPL and CL is higher in the secondary electron (SE2) detector. A combination of the two can e.g. be used to segment a CL/MPL interface with lower Pt particle content. This work shows the first measurement of the nano-roughness of a CL/MPL interface. Our roughness results are complementary to existing macroscopic measurements of GDEs and can be combined in order to virtually create an interface. As a next step we suggest reconstructing the CL/MPL interface of a CCM and GDL. It should be noted that such a reconstruction using FIBSEM tomography requires several steps: first, an assembled fuel cell needs to be embedded in epoxy in order to preserve it in the compressed state. Then the embedded sample needs to be sectioned in order to gain access to the interface. Once the sample is opened, ALD infiltration followed by FIB-SEM tomography can be performed. We are happy to share the reconstruction dataset on request.

\section{Acknowledgements}

We thank Jan Laube and Prof. Zacharias from the Laboratory for Nanotechnology for the atomic layer deposition of $\mathrm{ZnO}$ in the fuel cell electrode. This work was supported by the German
Federal Ministry of Education and Research (BMBF) [grant number 03SF0454C] and is part of the Gecko project.

\section{References}

1 H. Bajpai, M. Khandelwal, E. C. Kumbur and M. M. Mench, J. Power Sources, 2010, 195, 4196.

2 I. V. Zenyuk, E. C. Kumbur and S. Litster, J. Power Sources, 2013, 241, 379.

3 T. Chikahisa, Y. Tabe and K. Kadowaki, ASME Proceedings, 2012, p. 141.

4 F. E. Hizir, S. O. Ural, E. C. Kumbur and M. M. Mench, J. Power Sources, 2010, 195, 3463.

5 C.-Y. Liu and C.-C. Sung, J. Power Sources, 2012, 220, 348.

6 J. Zhang, PEM Fuel Cell Electrocatalysts and Catalyst Layers: Fundamentals and Applications, Springer, 2008.

7 H. Meng, Int. J. Hydrogen Energy, 2009, 34, 5488.

8 J. M. LaManna, V. Bothe James Jr, F. Y. Zhang and M. M. Mench, J. Power Sources, 2014, 271, 180.

9 J. Balach, F. Miguel, F. Soldera, D. F. Acevedo, F. Mücklich and C. A. Barbero, J. Microsc., 2012, 246, 274.

10 K. J. Lange, H. Carlsson, I. Stewart, P.-C. Sui, R. Herring and N. Djilali, Electrochim. Acta, 2012, 85, 322.

11 H. Schulenburg, B. Schwanitz, N. Linse, G. G. Scherer, A. Wokaun, J. Krbanjevic, R. Grothausmann and I. Manke, J. Phys. Chem. C, 2011, 115, 14236.

12 R. Singh, A. R. Akhgar, P. C. Sui, K. J. Lange and N. Djilali, J. Electrochem. Soc., 2014, 161, F415.

13 S. Thiele, T. Fürstenhaupt, D. Banham, T. Hutzenlaub, V. Birss, C. Ziegler and R. Zengerle, J. Power Sources, 2013, 228, 185.

14 S. Thiele, R. Zengerle and C. Ziegler, Nano Res., 2011, 4, 849. 15 S. Zils, M. Timpel, T. Arlt, A. Wolz, I. Manke and C. Roth, Fuel Cells, 2010, 10, 966.

16 E. A. Wargo, V. P. Schulz, A. Çeçen, S. R. Kalidindi and E. C. Kumbur, Electrochim. Acta, 2013, 87, 201.

17 E. A. Wargo, T. Kotaka, Y. Tabuchi and E. C. Kumbur, J. Power Sources, 2013, 241, 608.

18 E. A. Wargo, A. C. Hanna, A. Çeçen, S. R. Kalidindi and E. C. Kumbur, J. Power Sources, 2012, 197, 168.

19 H. Ostadi, P. Rama, Y. Liu, R. Chen, X. X. Zhang and K. Jiang, J. Membr. Sci., 2010, 351, 69.

20 A. Çeçen, E. A. Wargo, A. C. Hanna, D. M. Turner, S. R. Kalidindi and E. C. Kumbur, J. Electrochem. Soc., 2012, 159, B299.

21 S. Prass, S. Hasanpour, P. K. Sow, A. B. Phillion and W. Mérida, J. Power Sources, 2016, 319, 82.

22 T. Swamy, E. C. Kumbur and M. M. Mench, J. Electrochem. Soc., 2010, 157, B77.

23 M. Klingele, M. Breitwieser, R. Zengerle and S. Thiele, J. Mater. Chem. A, 2015, 3, 11239.

24 M. Breitwieser, M. Klingele, B. Britton, S. Holdcroft, R. Zengerle and S. Thiele, Electrochem. Commun., 2015, 60, 168.

25 N. Wehkamp, M. Breitwieser, A. Büchler, M. Klingele, R. Zengerle and S. Thiele, RSC Adv., 2016, 6, 24261. 
26 L. Holzer and M. Cantoni, Nanofabrication using focused ion and electron beams: Principles and applications, 2011, p. 410.

27 M. Ender, J. Joos, T. Carraro and E. Ivers-Tiffée, J. Electrochem. Soc., 2012, 159, A972.

28 S. Vierrath, F. Güder, A. Menzel, M. Hagner, R. Zengerle, M. Zacharias and S. Thiele, J. Power Sources, 2015, 285, 413.

29 S. Vierrath, L. Zielke, R. Moroni, A. Mondon, D. R. Wheeler, R. Zengerle and S. Thiele, Electrochem. Commun., 2015, 60, 176.

30 F. Güder, Y. Yang, U. M. Küçükbayrak and M. Zacharias, ACS Nano, 2013, 7, 1583.
31 MATLAB, R2014b, http://www.mathworks.de/, accessed, 28 January 2015.

32 M. D. Abràmoff, P. J. Magalhães and S. J. Ram, Biophotonics International, 2004, 11, 36.

33 J. F. Delerue, E. Perrier, Z. Y. Yu and B. Velde, Phys. Chem. Earth, 1999, 24, 639.

34 J. Becker, C. Wieser, S. Fell and K. Steiner, Int. J. Heat Mass Transfer, 2011, 54, 1360.

35 B. Standard, Method for the assessment of surface texture, British Standard Institution, London, 1972. 